\title{
Rule Mining Method for the Evaluation of Hotel Service Quality Based on Improved Genetic Algorithm
}

\author{
Yan Taishan ${ }^{+}$, Guo Guanqi, Li Wenbin \\ School of Information and Communication Engineering, Hunan Institute of Science and Technology, \\ Yueyang, Hunan, China 414000
}

\begin{abstract}
The market competition of modern hotel is becoming more and more intense, and the demand of hotel service quality is higher and higher. The regular evaluation of hotel service quality can improve its service quality and market competitive power. In the course of evaluating hotel service quality, the evaluation index system and evaluation knowledge rules are necessary. The factors which affect hotel service quality are analyzed and evaluation index system of hotel service quality is established in this study. An improved genetic algorithm is designed and a knowledge rule mining method for the evaluation of hotel service quality based on genetic algorithm is proposed. In the algorithm, new knowledge rules are generated by selection operator, adaptive crossover operator and adaptive mutation operator. Knowledge rules are evaluated by their accuracy, coverage and reliability. Experimental results show that this method can produce new knowledge individuals with higher fitness, so it is fully effective as a knowledge rule mining method.
\end{abstract}

Keywords: Knowledge rule mining; Evaluation of hotel service quality; Fitness of knowledge rule; Improve Genetic algorithm

\section{Introduction}

Knowledge rule mining means to extract useful knowledge rules from a large number of incomplete and noisy knowledge rules. There are several knowledge rule mining methods[1-3], such as machine learning, decision tree, neural network, fuzzy set theory, rough set, genetic algorithm etc... In these methods, genetic algorithm is widely applied depend on its high robustness and good global searching capability. In the evaluation index system of hotel service quality, there are many evaluation indexes, so, it is particularly effective to mine knowledge rules by genetic algorithm. In this study, an improved adaptive genetic algorithm is designed and used to mine evaluation knowledge rules of hotel service quality.

\section{Evaluation index system of hotel service quality}

Hotel service quality [4-7] is the service quality of intangible products, which is different from the quality of general physical products. The correct understanding of the characteristics of hotel service quality is the necessary condition to improve the hotel service quality. As a result of the particularity of hotel products, some characteristics of hotel service quality are formed as follows:

(1) Hotel service quality is a combination of tangible and intangible property. Equipment, facilities and daily articles provide use value for guests in physical form. They still exist in terms of physical form after being used. The labor form of the use value of service and its result will be disappeared after being used. It only leaves guests a satisfaction and feel.

(2) The composition of hotel service quality, which is composed of equipment and facilities, delicious food, quality of service, safety and environmental atmosphere.

\footnotetext{
+ Corresponding author. Tel.: +86 13117301054 .

E-mail address: yantaishan163@163.com
} 
(3) The evaluation for service quality of customer is one-off, it is difficult to repair afterwards. It requires the hotel management department of service quality must attach great importance to each specific service activities.

(4) The relationship between the contents of hotel service quality, which means hotel service quality is composed of some related specific service activities.

Therefore, the evaluation object of hotel service quality includes two aspects. The first is hardware service quality which is composed of facilities, equipment, supplies and environment. The second is software service quality which is composed of service items, service attitude, etiquette, service method, safety and hygiene.

Based on the extensive research of hotel service, we build an evaluation index system of hotel service quality. As we designed, the evaluation index system includes five primary indexes, they are hotel location, hotel service, hotel catering, guest room facilities and public environmental comfortless. Each primary index is broken down into a number of specific secondary indexes. These secondary indexes form the evaluation index system of hotel service quality, as Fig. 1 shows.

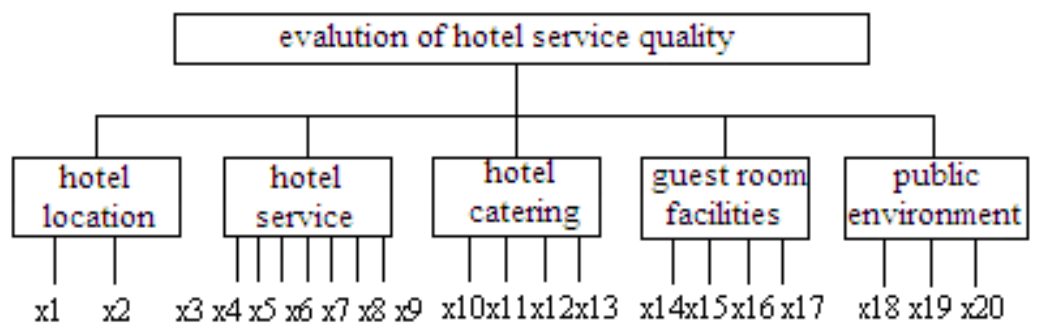

Fig.1: The evaluation index system of hotel service quality

Where, $\mathrm{x} 1$ and $\mathrm{x} 2$ denotes the hotel traffic and surrounding environment respectively. $\mathrm{x} 3, \mathrm{x} 4, \mathrm{x} 5 \mathrm{x} 6, \mathrm{x} 7, \mathrm{x} 8$ and $\mathrm{x} 9$ denotes the hotel shuttle service to the airport, baggage service, registration and checkout service, information service, booking service, housekeeping service and wake up service; respectively. $\mathrm{x} 10, \mathrm{x} 11, \mathrm{x} 12$ and $\mathrm{x} 13$ denotes the restaurant environment, restaurant service, food variety and quality and room delivery service respectively. $\mathrm{x} 14, \mathrm{x} 15, \mathrm{x} 16$ and $\mathrm{x} 17$ denotes the room sound insulation, guest bed supplies and appliances, room bathroom and room air conditioning respectively. x18, x19 and x20 denotes the lobby environment and facilities, recreational facilities, and overall health respectively.

\section{Knowledge rule representation method and improved genetic algorithm}

Knowledge rule representation is very important in data mining. There are several knowledge rule representation methods, such as predicate logic, production rule, semantic network, etc. Production rule representation is simple and clear, so, it has been the most widely used representation method of knowledge rule. Generally, production rule contains two parts, the first one is prerequisite and the second is conclusion. Prerequisite is the condition which must be provided with when the rule is implemented. Conclusion is the last result after the rule is implemented. A production rule can be described as follows:

$$
\text { IF E1(A1, A2 } \ldots, \text { Am ) } ・ \text { E2(A1, A2, .., Am ) } \bullet \ldots \cdot \text { En(A1, A2, ..Am) THEN C }
$$

Where, $\operatorname{Ei}(\mathrm{A} 1,2, \ldots, A n)(1 \leq i \leq n)$ denotes the prerequisite whose attributes are $\mathrm{Ai}(1 \leq \mathrm{i} \leq \mathrm{m}) . \mathrm{C}$ denotes the conclusion. - denotes the relation of AND.

Genetic algorithm[8-10] is a stochastic global searching algorithm used to solve complicated problems by simulating the biological evolutionary course of natural selection and natural inheritance. On account of the limitation of the simple genetic algorithm such as low convergence speed, premature convergence, etc., an improved adaptive genetic algorithm combined with individual consanguinity (called IAGA) is designed in this study. In order to avoid near consanguinity reproduction, two individuals need to detect the relationship after mating. If their similarity is too high, they need to be modified before crossing. The genetic operators of IAGA include selection operator, help operator, adaptive crossover operator and adaptive mutation operator. Fig.2 illustrates its working flow. 


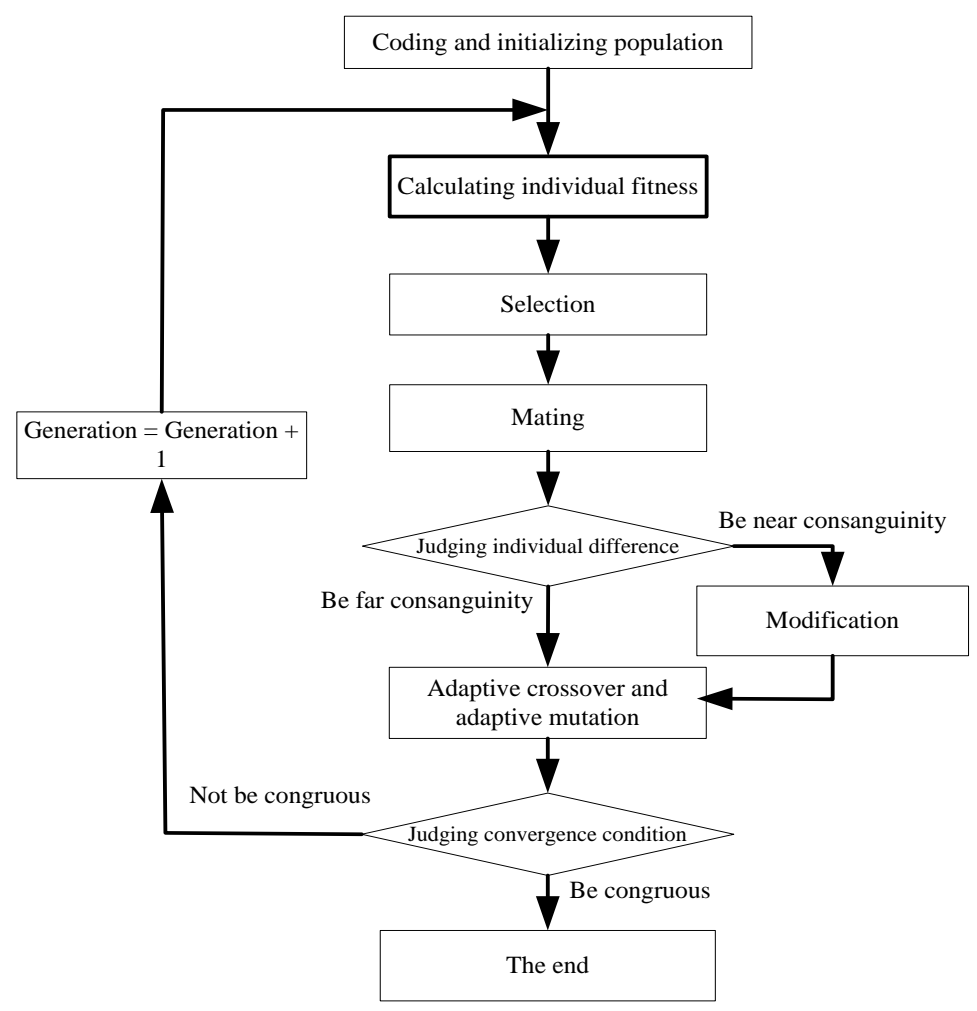

Fig.2: The working flow of IAGA

\section{Realization of genetic algorithm for mining knowledge rules of hotel service quality evaluation}

\subsection{Code Method of Knowledge Rules}

Genetic algorithm operates on code string only. For the problem of hotel service quality evaluation, it is easy to code knowledge rules by $0-1$ encoding mode. Each index and the evaluation conclusion have four grades, they are good(1), compared good (2), normal(3) and bad (4). They can be denoted by 00, 01, 10 and 11 respectively. This encoding mode is shown in Fig. 3.

\begin{tabular}{|c|c|c|c|c|c|c|c|c|}
\hline Flag $_{1}$ & $U_{1}$ & $\ldots$ & Flag $_{\mathrm{i}}$ & $U_{\mathrm{i}}$ & $\ldots$ & Flag $_{\mathrm{n}}$ & $U_{\mathrm{n}}$ & $V$ \\
\hline
\end{tabular}

Fig.3: The code structure of knowledge rule individual

Where, $\mathrm{Ui}$ is the code of the attributes of number i prerequisite. Flagi is the BOOL flag bit, it denotes if the number $\mathrm{i}$ condition is contained in rule prerequisites. Flagi=1 means the number i condition is contained in rule prerequisites. Flagi $=0$ means the number $i$ condition is not contained in rule prerequisites. $\mathrm{V}$ is the code of conclusion attribute value.

\subsection{Evaluation Method of Knowledge Rules.}

The linear combination method is used to evaluate the knowledge rules in our study. Knowledge rules are evaluated by three important factors. They are accuracy, coverage and reliability. Suppose $\mathrm{U}$ is a test data set, e is an instance (elements) of $\mathrm{U}$. The expression of these three parameters can be defined as:

$$
\begin{array}{ll}
\text { - Accuracy: } & A\left(r_{i}\right)=\frac{\left|\Omega_{1 r_{i}}^{U}\right|}{\left|\Omega_{1 r_{i}}^{U}\right|+\left|\Omega_{2 r_{i}}^{U}\right|} \\
\text {-Coverage: } & B\left(r_{i}\right)=\frac{\left|\Omega_{1 r_{i}}^{U}\right|+\left|\Omega_{2 r_{i}}^{u}\right|}{\max \left(\left|\Omega_{1 r_{j}}^{U}\right|+\left|\Omega_{2 r_{j}}^{U}\right|\right.} \\
\text { - Reliability: } & C\left(r_{i}\right)=\frac{1+\left(T_{r_{i}}-1\right) / T_{C}}{T_{r_{i}}}
\end{array}
$$

Where, $\Omega_{1 r_{i}}^{U}$ is a subset of $\mathrm{U}$, each of its elements (instances) match knowledge rule ri that need to be evaluated. $\Omega_{2 r_{i}}^{U}$ is another subset of $\mathrm{U}$, each of its elements (instances) only match knowledge rule ri that 
need to be evaluated with prerequisite. $T_{r_{i}}$ denotes the theoretical number of rules in the knowledge rule base that match the knowledge rule ri with complete condition. $T_{C}$ denotes the number of conclusion in the knowledge rule base.

\subsection{Genetic operators.}

In IAGA, key operators include selection operator, adaptive crossover operator and adaptive mutation operator. Selection operator is often realized by the roulette wheel selection method. In order to maintain the diversity of population and ensure the convergence of genetic algorithm, in the course of crossover and mutation, the probability of crossover and the probability of mutation are adjusted adaptively according to the following formulas:

$$
\begin{gathered}
P_{c}=\left\{\begin{array}{cr}
P_{c \text { min }}+\frac{P_{c \text { max }}-P_{c \min }}{1+\exp \left(-\left(\frac{f^{\prime}-f_{\text {avg }}}{f_{\text {max }}-f_{\text {avg }}}\right)\right)}, & f^{\prime} \geq f_{\text {avg }} \\
P_{c \max }, & f^{\prime}\left\langle f_{\text {avg }}\right.
\end{array}\right. \\
P_{m}=\left\{\begin{array}{cl}
P_{m \text { min }}+\frac{P_{m \text { max }}-P_{m \text { min }}}{1+\exp \left(-\left(\frac{f-f_{\text {avg }}}{f_{\text {max }}-f_{\text {avg }}}\right)\right)}, & f \geq f_{\text {avg }} \\
P_{m \text { max }}, & f<f_{\text {avg }}
\end{array}\right.
\end{gathered}
$$

Where, $P_{c \min }$ and $P_{c \text { max }}$ denotes the lower limit and the upper limit of the probability of crossover respectively, $f_{\max }$ denotes the maximal fitness of the population, $f_{\text {avg }}$ denotes the average fitness of the population, $f^{\prime}$ denotes the higher fitness of the two crossover individuals, $f$ denotes the fitness of mutation individual.

\section{Example}

Table 1 is a knowledge rule base(part knowledge rules) of the evaluation of hotel service quality. And it is regarded as the test data set. The code and fitness of the knowledge rules in table 1 are shown in table 2. C in table 1 denotes the evaluation conclusion. A in table 1 denotes the corresponding index value can be arbitrary.

\begin{tabular}{|c|c|c|c|c|c|c|c|c|c|c|c|c|c|c|c|c|c|c|c|c|c|}
\hline ID & $\mathrm{x} 1$ & $\mathrm{x} 2$ & x3 & $\mathrm{x} 4$ & x5 & x6 & $\mathrm{x} 7$ & $\mathrm{x} 8$ & $\mathrm{x} 9$ & x10 & $\mathrm{x} 11$ & $\mathrm{x} 12$ & x13 & $\mathrm{x} 14$ & $\times 15$ & $\mathrm{x} 16$ & $\mathrm{x} 17$ & $7 \times 18$ & x19 & $\mathrm{x} 20$ & C \\
\hline 1 & 1 & 1 & 2 & 1 & 1 & 1 & 1 & 1 & 1 & 1 & 2 & 1 & 1 & 1 & 2 & 1 & 1 & 2 & 1 & 1 & 1 \\
\hline 2 & 1 & 1 & 1 & 1 & 1 & 1 & 1 & 1 & 2 & 1 & 1 & 1 & 1 & 1 & 1 & 1 & 1 & 1 & 1 & 1 & 1 \\
\hline 3 & 1 & 1 & 1 & 1 & 2 & 1 & 1 & 2 & 2 & 1 & 1 & 1 & 2 & 1 & 1 & 1 & 2 & 2 & 1 & 1 & 2 \\
\hline 4 & 1 & 2 & 1 & 1 & 1 & 2 & 1 & 1 & 1 & 1 & 1 & 2 & 2 & 1 & 1 & 2 & 1 & 1 & 2 & 1 & 2 \\
\hline 5 & 2 & 2 & 2 & 2 & 2 & 3 & 1 & 2 & 1 & 2 & 3 & 3 & 3 & 2 & 2 & 2 & 3 & 1 & 2 & 1 & 3 \\
\hline 6 & 1 & 1 & 2 & 2 & 3 & 1 & 3 & 2 & 1 & 2 & 3 & 1 & 2 & 2 & 1 & 2 & 3 & 1 & 3 & 2 & 3 \\
\hline 7 & 2 & 2 & 3 & 4 & 4 & 3 & 4 & A & 3 & 2 & 3 & 3 & 4 & 2 & 3 & 4 & 4 & 3 & 4 & A & 4 \\
\hline 8 & 3 & 3 & 2 & 2 & 3 & 4 & 3 & 4 & 3 & 3 & 4 & 4 & A & 3 & 3 & 4 & 3 & 4 & 3 & 3 & 4 \\
\hline
\end{tabular}

Table 1 Knowledge rule base of hotel service quality evaluation

Table 2 The code and fitness of the knowledge rules in table 1

\begin{tabular}{ccc}
\hline ID & Code & Fitness \\
\hline 1 & 10110010010010010010110010110010110010010010000 & 0.957504 \\
2 & 10010010010010010010110110010010010010010010100 & 0.957504 \\
3 & 10110110110010010110110010010011010010110100001 & 0.907429 \\
4 & 10010010010010010000010010010010010010010010100 & 0.899652 \\
5 & 10110010010010010010011110010010110010110111001 & 0.957504 \\
6 & 10110010010010011011010010010010110011010110001 & 0.957504 \\
7 & 101101110101111110000110111111111111010111100011 & 0.818346 \\
8 & 11010110110111010111011010110111011100011011010 & 0.897438 \\
\hline
\end{tabular}

After genetic algorithm iterates 2 times, two valid new knowledge rules are generated. Their fitness is 0.901245 and 0.957504 . As table 3 and table 4 shows. This two valid new knowledge rules will be added to the knowledge rule base of hotel service quality evaluation. And the knowledge rule base is updated. 
Table 3 The code and fitness of new knowledge rules

\begin{tabular}{rcc}
\hline ID & Code & Fitness \\
\hline 9 & 10010110010010010100010010010110011010010110101 & 0.901245 \\
10 & 10010010110010010010110010110010010010010110000 & 0.957504 \\
\hline
\end{tabular}

Table 4 The new knowledge rules

\begin{tabular}{c|cccccccccccccccccccc|c}
\hline ID & \multicolumn{1}{|c}{$\mathrm{x} 1$} & $\mathrm{x} 2$ & $\mathrm{x} 3$ & $\mathrm{x} 4$ & $\mathrm{x} 5$ & $\mathrm{x} 6$ & $\mathrm{x} 7$ & $\mathrm{x} 8$ & $\mathrm{x} 9$ & $\mathrm{x} 10$ & $\mathrm{x} 11$ & $\mathrm{x} 12$ & $\mathrm{x} 13$ & $\mathrm{x} 14$ & $\mathrm{x} 15$ & $\mathrm{x} 16$ & $\mathrm{x} 17$ & $\mathrm{x} 18$ & $\mathrm{x} 19$ & $\mathrm{x} 20$ & $\mathrm{C}$ \\
\hline 1 & 2 & 2 & 2 & 2 & 2 & 3 & 1 & 2 & 1 & 2 & 3 & $\mathrm{~A}$ & 3 & 2 & 2 & 2 & 3 & 1 & 2 & 1 & 3 \\
2 & 1 & 1 & 2 & 1 & 1 & 1 & 1 & 1 & 1 & 1 & 2 & 1 & 1 & 1 & 2 & 1 & 1 & 2 & 1 & 1 & 1 \\
\hline
\end{tabular}

\section{Conclusion}

The index system of hotel service quality evaluation includes five primary indexes, they are hotel location, hotel service, hotel catering, guest room facilities and public environmental comfortless. An improved adaptive genetic algorithm combined with individual consanguinity is designed and used to mine the knowledge rules. A knowledge rule mining method for the evaluation of hotel service quality based on improved genetic algorithm is proposed. The experimental results show that better new knowledge rules can be generated by this method. The improved genetic algorithm is fully valid in mining knowledge rules. It will help us to evaluate hotel service quality fairly and objectively.

\section{Acknowledgments}

Support From the Natural Science Foundation of Hunan Province under Grant No.2017JJ2107, the Science and Technology Program of Hunan Province under Grant No. 2016TP1021 and the Graduate Course Construction Project "Intelligent Information Processing" of Hunan Institute of Science and Technology under Grant No.2015 is gratefully acknowledged.

\section{References}

[1] Diao Lili. Data mining and Combination Learning. Computer Science, 2001, 28(7):73 78

[2] Dasarathy, B.V Nearest Neighbor(NN)Norms. NN Pattern Classification Techniques. Washington, D.C.: IEEE Computer Society, 1991

[3] ZIARKO W. Rough sets, Fuzzy Sets and Knowledge Discovery. New York: Springer-Verlag, 1994

[4] Zhang Huanyong, Li Feng, Zhang Xia. The evaluation and improvement of Economic Hotel service quality based on customer satisfaction. Commercial Age,2013(36):68-69

[5] $\mathrm{Hu}$ Bin. On the construction of service quality evaluation system for small and medium sized single Hotel. Shopping modernization, 2016(21):113-114.

[6] Liu Ying, Xue Yaowen. Construction of hotel service quality evaluation system. Mechanical management and development, 2009, 24(4):137-139.

[7] Zhang Y. Theoretical Analysis and Cultivation Countermeasure of Customer Loyalty in Hotel Enterprise. Canadian Social Science, 2006, 2(4):104-107.

[8] Li MinQiang, Kou JiSong, Lin Dan, Li ShuQuan, Basic principle and application of genetic algorithm, Science publishing company, Beijing, 2002

[9] Wang Xiaoping, Cao Liming, Genetic algorithm - theory, application and software realization, Xi'an Jiao tong University Press, Xi'an, 2002

[10] Thomas Strassner, Markus Busold, Wolfgang A. Herrmann, MM 3 parametrization of four- and five- coordinated rhenium complexes by a genetic algorithm. Journal of Computational chemistry, Vol.23, 2002. 